\title{
Influence des paramètres rhéologiques sur les limites de formage des tôles minces
}

\author{
Hassan Fatmaoui ${ }^{\mathrm{a}}$, Rachid Mesrar et Jamal Chaoufi \\ Laboratoire de Mécanique et Milieux Continus, Université Ibn Zohr, Faculté des Sciences, Agadir, Maroc
}

Reçu le 10 juin 2005, accepté le 20 juin 2006

\begin{abstract}
Résumé - Dans ce travail, la striction localisée des tôles minces est étudiée en utilisant une analyse de localisation type Marciniak-Kuczynski. Un nouveau critère de plasticité orthotrope est utilisé pour déterminer les limites de formage pour des chemins linéaires de déformation. Par ailleurs l'influence des paramètres rhéologiques du matériau sur les courbes limites de formage (CLF) a été étudiée et analysée ainsi que celui de la surface de charge.
\end{abstract}

Mots clés : Critère de plasticité / analyse de localisation / courbes limites de formage

\begin{abstract}
Influence of material parameters on the forming limits of sheet metals. The forming limits in biaxially stretched sheets are analysed by using a Marciniak-Kuczynski localization approach with a new plane-stress yield criterion. The limits to ductility depend on the strain-hardening capacity and the strain-rate sensitivity of the material. In biaxially stretched sheets, an additional material parameter that controls the forming limits is the yield locus curvature. The effect to the material parameters is analysed with particular attention to the yield surface shape on the forming limits diagrams.
\end{abstract}

Key words: Plasticity criterion / localization analyses / forming limit diagrams

\section{Introduction}

Les procédés de formage des matériaux, notamment l'emboutissage des tôles, correspondent à des processus complexes où intervient un grand nombre de paramètres difficiles à cerner et à maîtriser. Par là même, il va de soi qu'une connaissance approfondie du matériau et de sa formabilité est nécessaire pour l'utilisation dans des conditions optimales et pour rationaliser les procédés de mise en forme. Les limites imposées au formage des tôles par le développement d'une zone de striction localisée ont été particulièrement étudiées et modélisés à partir du concept de courbe limite de formage (CLF). Les courbes sont définies pour des trajectoires de déformation rectilignes ou quasi-rectilignes, et sont obtenues en portant dans les axes des déformations principales, dans le plan de la tôle, les couples de valeurs $\left(\varepsilon_{1}^{*}, \varepsilon_{2}^{*}\right)$ correspondant respectivement à la plus grande et à la plus petite des déformations principales à striction.

Une analyse de localisation de la déformation en présence d'un défaut d'épaisseur est développée dans ce travail. Cette analyse (type MK) [1] est utilisée afin d'obtenir les courbes limites de formage (CLF) des tôles.

\footnotetext{
a Auteur correspondant : hfatmaoui@caramail.com
}

Par ailleurs des simulations numériques nous ont permis d'étudier l'influence de la capacité d'écrouissage du matériau, de l'anisotropie, de la sensibilité à la vitesse de déformation ainsi que la taille du défaut sur la forme et le niveau des limites de formage. L'influence de la forme de la surface d'écoulement a principalement été étudiée pour le rôle capital qu'il joue notamment pour des trajectoires dans le domaine de l'expansion.

\section{Analyse de localisation de la déformation}

\subsection{Formulation du problème de localisation}

L'analyse de localisation est basée sur l'hypothèse de l'existence d'une bande initiale de sous-épaisseur par rapport aux directions principales de contraintes (Fig. 1). La taille initiale de ce défaut est définie par :

$$
f=\frac{h^{\mathrm{b}}(0)-h^{\mathrm{n}}(0)}{h^{\mathrm{b}}(0)}
$$

où $h(t)$ est l'épaisseur à l'instant $t$, et les indices supérieurs $\mathrm{b}$ et $\mathrm{n}$ se référent respectivement à la zone homogène et à 


\section{Nomenclature}

\begin{tabular}{|c|c|}
\hline$K, \varepsilon_{0}, \dot{\varepsilon}_{0}$ & constantes du matériau \\
\hline$k, A, B$ & paramètres du modèle \\
\hline$N$ & coefficient d'écrouissage \\
\hline$M$ & $\begin{array}{l}\text { coefficient de sensibilité à la vitesse } \\
\text { de déformation }\end{array}$ \\
\hline$R$ & coefficient de Lankford \\
\hline$f$ & taille initiale du défaut \\
\hline$h$ & épaisseur de la tôle \\
\hline$t$ & temps \\
\hline$\theta$ & $\begin{array}{l}\text { angle polaire d'un point de la surface } \\
\text { de charge normalisée }\end{array}$ \\
\hline $\mathrm{g}(\theta)$ & $\begin{array}{l}\text { rayon polaire d'un point de la surface } \\
\text { de charge normalisée }\end{array}$ \\
\hline$\alpha$ & $\sigma_{\mathrm{nt}}^{\mathrm{n}} / \sigma_{\mathrm{nn}}^{\mathrm{n}}$ rapport de contraintes \\
\hline$\varepsilon$ & déformation logarithmique \\
\hline $\bar{\varepsilon}$ & déformation équivalente \\
\hline$\dot{\bar{\varepsilon}}$ & vitesse de déformation équivalente \\
\hline$\Delta \varepsilon$ & incrément de déformation \\
\hline$\rho$ & $\dot{\varepsilon}_{2} / \dot{\varepsilon}_{1}$ rapport des vitesses de déformation \\
\hline$\sigma$ & contrainte de Cauchy \\
\hline$\sigma_{\mathrm{b}}$ & contrainte de traction équibiaxiale \\
\hline$\sigma_{\mathrm{tp}}$ & contrainte majeure en traction plane \\
\hline$\sigma_{\mathrm{u}}$ & contrainte de traction uniaxiale \\
\hline $\bar{\sigma}$ & contrainte équivalente \\
\hline$\psi$ & inclinaison de la bande \\
\hline$\psi(0)$ & inclinaison initiale de la bande \\
\hline$G$ & $\begin{array}{l}\text { longueur du rayon polaire d'un point } \\
\text { de la surface de charge dans les axes }\left(x_{1}, x_{2}\right) \text {. }\end{array}$ \\
\hline \multicolumn{2}{|l|}{ Indices } \\
\hline 1,2 & directions des contraintes principales \\
\hline $\mathrm{n}, \mathrm{t}$ & $\begin{array}{l}\text { directions normale et tangentielle } \\
\text { de la bande }\end{array}$ \\
\hline \multicolumn{2}{|c|}{ Exposants } \\
\hline $\mathrm{b}$ & zone homogène (bulk) \\
\hline $\mathrm{n}$ & zone du défaut (neck) \\
\hline
\end{tabular}

la zone de sous-épaisseur. Le matériau est rigide viscoplastique. Il obéit à la théorie de l'écoulement avec écrouissage isotrope. En supposant en outre un comportement à isotropie plane, les directions principales de contraintes et de vitesse de déformation coïncident, dans l'une ou l'autre des zones.

Les directions principales $(1,2)$ dans la zone homogène sont supposées fixes par rapport à un système d'axes matériels. Dans le cas général où la bande de sous-épaisseur n'est pas dirigée selon une direction principale, elle tourne au cours de la déformation, et l'angle $\psi^{\mathrm{b}}=(\mathrm{n}, 1)=(\mathrm{t}, 2)$ définissant l'inclinaison de la bande par rapport aux directions principales dans la zone homogène est lié à sa valeur initiale $\psi^{\mathrm{b}}(0)$ par :

$$
\tan \psi^{\mathrm{b}}=\exp \left(\varepsilon_{1}^{\mathrm{b}}-\varepsilon_{2}^{\mathrm{b}}\right) \tan \psi^{\mathrm{b}}(0)
$$

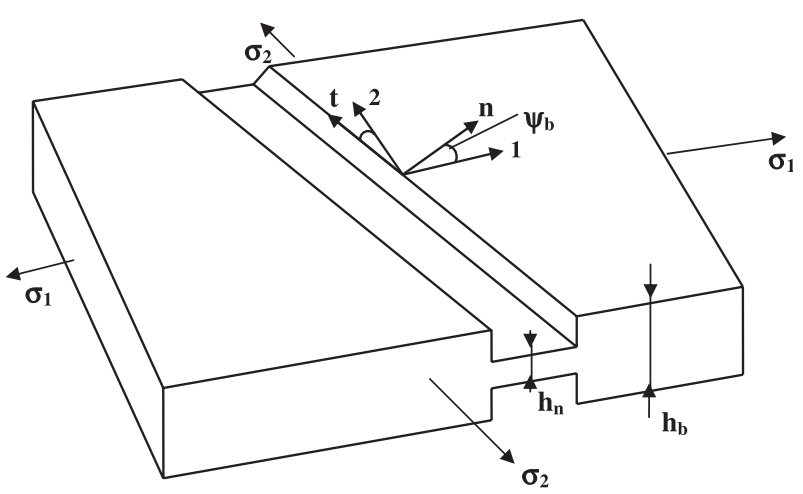

Fig. 1. Schéma d'un défaut d'épaisseur dans une tôle.

où $\varepsilon_{1}^{\mathrm{b}}$ et $\varepsilon_{2}^{\mathrm{b}}$ désignent respectivement la plus grande et la plus petite des déformations principales dans le plan de la tôle. Les équations de base sont fournies par les équations d'équilibre :

$$
\begin{aligned}
\sigma_{\mathrm{nn}}^{\mathrm{b}} h^{\mathrm{b}} & =\sigma_{\mathrm{nn}}^{\mathrm{n}} h^{\mathrm{n}} \\
\sigma_{\mathrm{nt}}^{\mathrm{b}} h^{\mathrm{b}} & =\sigma_{\mathrm{nt}}^{\mathrm{n}} h^{\mathrm{n}}
\end{aligned}
$$

La condition de compatibilité des déformations est :

$$
\dot{\varepsilon}_{\mathrm{tt}}^{\mathrm{b}}=\dot{\varepsilon}_{\mathrm{tt}}^{\mathrm{n}}
$$

et les expressions des contraintes et des vitesses de déformation dans les axes $(n, t)$ :

$$
\begin{aligned}
\sigma_{\mathrm{nn}} & =\sigma_{1} \cos ^{2} \psi+\sigma_{2} \sin ^{2} \psi \\
\sigma_{\mathrm{nt}} & =-\left(\sigma_{1}-\sigma_{2}\right) \cos \psi \sin \psi \\
\dot{\varepsilon}_{\mathrm{tt}} & =\dot{\varepsilon}_{1} \sin ^{2} \psi+\dot{\varepsilon}_{2} \cos ^{2} \psi
\end{aligned}
$$

où $\sigma_{i j}$ et $\dot{\varepsilon}_{i j}$ sont respectivement les composantes des contraintes de Cauchy et des vitesses de déformation. Dans le cas où la bande n'est pas dirigée selon la direction de plus petite contrainte principale (c'est-à-dire dans le domaine du rétreint, où l'angle $\psi^{\mathrm{b}}(0)$ minimisant les déformations limites est différent de zéro), les directions principales de contraintes et la vitesse de déformation dans la zone de sous-épaisseur tournent au cours de la déformation. Dans la mise en équation développée ciaprès, l'orientation $\psi^{\mathrm{n}}$ de la bande par rapport aux directions principales dans la zone de sous-épaisseur est prise explicitement comme inconnue du problème de localisation. Les équations (6)-(8) seront donc utilisées dans l'une ou l'autre zone.

\subsection{Surface de plasticité et loi d'écoulement associée}

Les équations proposées par Ferron et al. [2,3] pour décrire une surface de charge orthotrope sont utilisées ici dans le cas d'un matériau à isotropie plane. L'expression de la surface de charge peut alors être écrite sous la forme :

$$
f_{\mathrm{c}}=\phi\left(\sigma_{1}, \sigma_{2}\right)-\sigma=0
$$

où $\sigma$ est une mesure de la contrainte équivalente, prise ici comme étant la contrainte d'écoulement en traction 


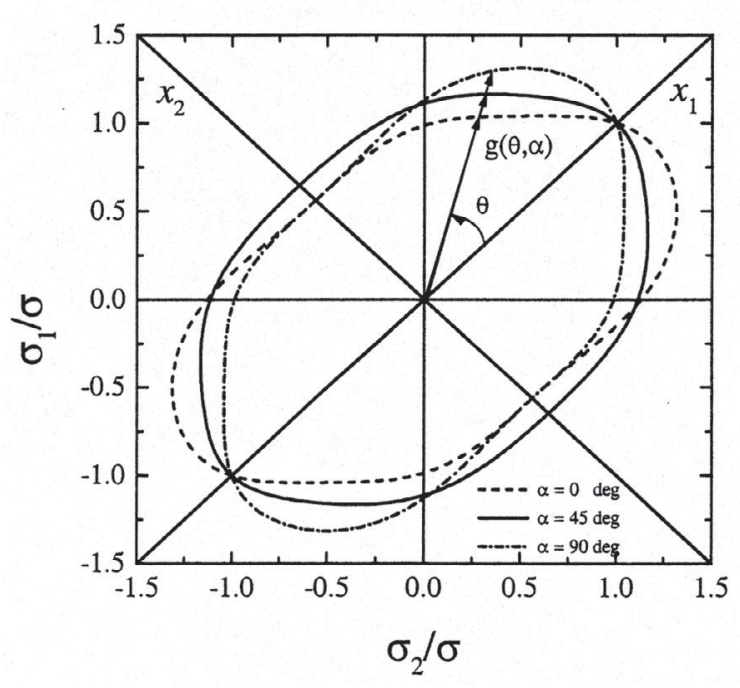

Fig. 2. Représentation paramétrique de la surface de charge dans l'espace des contraintes principales.

équibiaxiale. En utilisant le changement de variables défini par : $x_{1}=\left(\sigma_{1}+\sigma_{2}\right) / 2$ et $x_{2}=\left(\sigma_{1}-\sigma_{2}\right) / 2$, l'équation de la surface peut être ré-exprimée :

$$
f_{\mathrm{c}}=\psi\left(x_{1}, x_{2}\right)-\sigma=0
$$

Une représentation paramétrique de la surface de charge définie par :

$$
\begin{aligned}
& x_{1}=x_{1}(\theta, \sigma)=G(\theta, \sigma) \cos \theta \\
& x_{2}=x_{2}(\theta, \sigma)=G(\theta, \sigma) \sin \theta
\end{aligned}
$$

est finalement adoptée, dans laquelle $G(\theta, \sigma)$ représente la longueur du rayon polaire d'un point de la surface de charge représentée dans les axes $\left(x_{1}, x_{2}\right)$, et le paramètre $\theta$ est l'angle polaire associé. Dans l'hypothèse d'une expansion isotrope des surfaces de charge, $\phi\left(\sigma_{1}, \sigma_{2}\right)$ et $\psi\left(x_{1}, x_{2}\right)$ sont des fonctions homogènes de degré un, et $G(\theta, \sigma)$ s'exprime par :

$$
G(\theta, \sigma)=\sigma g(\theta)
$$

où $g(\theta)$ représente la longueur du rayon polaire d'un point de la surface de charge normalisée par $\sigma$. Le principe de cette représentation paramétrique est illustré sur la figure 2 .

En utilisant les relations précédentes on obtient en particulier :

$$
\begin{aligned}
& \sigma_{1}=\sigma g \sqrt{2} \sin (\theta+\pi / 4) \\
& \sigma_{2}=\sigma g \sqrt{2} \cos (\theta+\pi / 4)
\end{aligned}
$$

La loi de normalité permet d'exprimer les vitesses de déformation plastique [2] :

$$
\begin{aligned}
& \dot{\varepsilon}_{1}=\frac{g \sin (\theta+\pi / 4)-g^{\prime} \cos (\theta+\pi / 4)}{\sqrt{2} g^{2}} \dot{\varepsilon} \\
& \dot{\varepsilon}_{2}=\frac{g \cos (\theta+\pi / 4)+g^{\prime} \sin (\theta+\pi / 4)}{\sqrt{2} g^{2}} \dot{\varepsilon}
\end{aligned}
$$

où $\dot{\varepsilon}$ est la vitesse de déformation plastique équivalente conjuguée de $\sigma$. En particulier, le rapport de vitesses de déformation $\rho=\dot{\varepsilon}_{2} / \dot{\varepsilon}_{1}$ est donné par :

$$
\rho=\frac{g \cos (\theta+\pi / 4)+g^{\prime} \sin (\theta+\pi / 4)}{g \sin (\theta+\pi / 4)-g^{\prime} \cos (\theta+\pi / 4)}
$$

Une expression de la fonction $g(\theta)$ permettant d'obtenir un bon ajustement de résultats expérimentaux [2] est obtenue à partir d'une extension du critère isotrope de [4], défini par :

$$
\begin{aligned}
(1-k) g(\theta)^{-6}=\left(\cos ^{2} \theta\right. & \left.+A \sin ^{2} \theta\right)^{3} \\
& -k \cos ^{2} \theta\left(\cos ^{2} \theta-B \sin ^{2} \theta\right)^{2}
\end{aligned}
$$

où $A$ et $B$ sont des constantes positives $(A=3$ et $B=9$ pour le critère de Drucker).

Le critère quadratique de Hill [5] est également retrouvé avec $k=0$ et $A=1+2 R$, où $R$ est le coefficient de Lankford.

La fonction de charge $f_{c}$ préalablement définie est finalement utilisée comme potentiel plastique pour un matériau viscoplastique dont la loi de comportement équivalente est exprimée sous une forme multiplicative :

$$
\sigma=K\left(\varepsilon_{0}+\varepsilon\right)^{N}\left(\frac{\dot{\varepsilon}}{\dot{\varepsilon}_{0}}\right)^{M}
$$

où $\dot{\varepsilon}_{0}$ est une vitesse de déformation de référence, $N$ est l'exposant d'écrouissage, $M$ est le coefficient de sensibilité à la vitesse de déformation, $K$ et $\varepsilon_{0}$ sont des constantes.

Un changement de définition des contraintes, vitesse de déformation et déformation équivalentes est obtenu très facilement à partir des équations (13). En désignant par $\tilde{\sigma}, \dot{\tilde{\varepsilon}}$ et $\tilde{\varepsilon}$ les quantités équivalentes identifiées en traction plane, on obtient : $\tilde{\sigma} / \sigma=\dot{\varepsilon} / \dot{\tilde{\varepsilon}}=\varepsilon / \tilde{\varepsilon}=P$, où le rapport $P$ entre les contraintes d'écoulement en traction plane $\left(\sigma_{\mathrm{tp}}\right)$ et en traction équibiaxiale $\left(\sigma_{\mathrm{b}}\right)$ est donné par : $P=g\left(\sigma_{\mathrm{tp}}\right) \sqrt{2} \sin \left(\sigma_{\mathrm{tp}}+\pi / 4\right)$.

Si l'on se réfère à l'usage habituel des quantités équivalentes $\sigma_{\mathrm{u}}, \dot{\varepsilon}_{\mathrm{u}}$ et $\varepsilon_{\mathrm{u}}$ identifiées en traction uniaxiale, on obtient : $\sigma_{\mathrm{u}} / \sigma=\dot{\varepsilon} / \dot{\varepsilon}_{\mathrm{u}}=\varepsilon / \varepsilon_{\mathrm{u}}=\sqrt{2} g(\pi / 4)$

Le tableau 1 qui suit précise les valeurs numériques de $P=\sigma_{\mathrm{tp}} / \sigma_{\mathrm{b}}$ en fonction du coefficient de Lankford $R$.

Tableau 1. Variation de $P$ en fonction de $R$.

\begin{tabular}{ccc}
\hline & \multicolumn{2}{c}{$P=\sigma_{\mathrm{tp}} / \sigma_{\mathrm{b}}$} \\
\hline Coefficient & Critère & Modèle \\
de Lankford $R$ & quadratique de Hill & \\
\hline 0,5 & 1,2246 & 1,1343 \\
1,0 & 1,1546 & 1,0880 \\
2,0 & 1,0952 & 1,0598 \\
4,0 & 1,0532 & 1,0412 \\
\hline
\end{tabular}

\section{Résolution des équations de localisation}

La résolution du système intégro-différentiel représentant les équations de localisation s'effectue par 
une méthode de Runge-Kutta du quatrième ordre associée à une méthode de Newton.

Les calculs se font de façon incrémentale, en utilisant une procédure itérative implicite où le système d'équations suivant, déduit de (3)-(5), doit être satisfait à la fin de l'incrément :

$$
\begin{aligned}
\frac{\sigma_{\mathrm{nt}}^{\mathrm{n}}}{\sigma_{\mathrm{nn}}^{\mathrm{n}}} & =\alpha^{\mathrm{b}} \\
\sigma_{\mathrm{nn}}^{\mathrm{n}} h^{\mathrm{n}} & =F_{\mathrm{n}}^{\mathrm{b}} \\
\Delta \varepsilon_{\mathrm{tt}}^{\mathrm{n}} & =\Delta \varepsilon_{\mathrm{tt}}^{\mathrm{b}}
\end{aligned}
$$

où $\alpha^{\mathrm{b}}=\sigma_{\mathrm{nt}}^{\mathrm{b}} / \sigma_{\mathrm{nn}}^{\mathrm{b}}$ et $F_{n}^{\mathrm{b}}=\sigma_{\mathrm{nn}}^{\mathrm{b}} h^{\mathrm{b}}$ est la force normale à la bande. Le chemin de déformation imposé dans la zone homogène peut être caractérisé par l'angle $\theta^{\mathrm{b}}$ sur la surface de charge (Éq. (15)) de façon équivalente par le rapport de vitesses de déformation $\rho^{\mathrm{b}}$. L'incrément de déformation équivalente $\Delta \varepsilon^{\mathrm{b}}$ étant fixé, tous les incréments pour la zone homogène peuvent alors être déterminés. Les incréments des déformations principales $\Delta \varepsilon_{1}^{\mathrm{b}}$ et $\Delta \varepsilon_{2}^{\mathrm{b}}$ sont calculés à l'aide de (14), et l'incrément de déformation en épaisseur $\Delta \varepsilon_{3}^{\mathrm{b}}$ est déterminé par la condition d'incompressibilité plastique. L'incrément d'épaisseur $\Delta h^{\mathrm{b}}=h^{\mathrm{b}} \Delta \varepsilon_{3}^{\mathrm{b}}$ est donc connu, et toutes les déformations peuvent être réactualisées à la fin de l'incrément. La nouvelle inclinaison de la bande est calculée en utilisant (2), et l'incrément $\Delta \varepsilon_{\mathrm{tt}}^{\mathrm{b}}$ tangent à la bande est déterminé par (8). La nouvelle contrainte équivalente $\sigma^{\mathrm{b}}$ est donnée par (17), les nouvelles contraintes $\sigma_{1}^{\mathrm{b}}$ et $\sigma_{2}^{\mathrm{b}}$ sont déduites de $(13), \sigma_{\mathrm{nn}}^{\mathrm{b}}$ et $\sigma_{\mathrm{nt}}^{\mathrm{b}}$ sont obtenues à partir de (6) et (7).

Les membres de droite des équations (18)-(20) sont finalement connus, ces équations représentent un système non-linéaire avec $\theta^{\mathrm{n}}, \psi^{\mathrm{n}}$ et $\Delta \varepsilon^{\mathrm{n}}$ comme inconnues. La limite de déformation est supposée atteinte lorsque le rapport des incréments de déformations équivalentes $\Delta \varepsilon^{\mathrm{n}} / \Delta \varepsilon^{\mathrm{b}}$ devient plus grand qu'une valeur prédéterminée, qui a été prise égale à 50 . Dans le domaine du rétreint, l'angle $\psi^{\mathrm{b}}(0)$ doit être déterminé de façon à minimiser les déformations limites. Cet angle est nul dans le domaine de l'expansion pour un matériau à isotropie planaire.

\section{Résultats et discussions}

De nombreuses études [5-10] sont relatives à la taille initiale du défaut $(f)$ et aux paramètres caractérisant l'écrouissage (N) [11-14], La sensibilité à la vitesse de déformation $(M)$ [15] et la contrainte équivalente (ou la forme de la surface de charge) ont été publiées. On peut noter tout d'abord que la limite de formage est la plus basse en terme de déformation équivalente en traction plane. Pour une taille de défaut donnée et pour une loi d'écrouissage donnée en traction plane, la déformation limite $\varepsilon_{1 \text { tp }}^{*}$ en traction plane ne dépend pas de la surface de charge considérée. Ces propriétés proviennent du fait que la zone homogène et la zone de sous-épaisseur suivent alors le même trajet de déformation. Par contre,

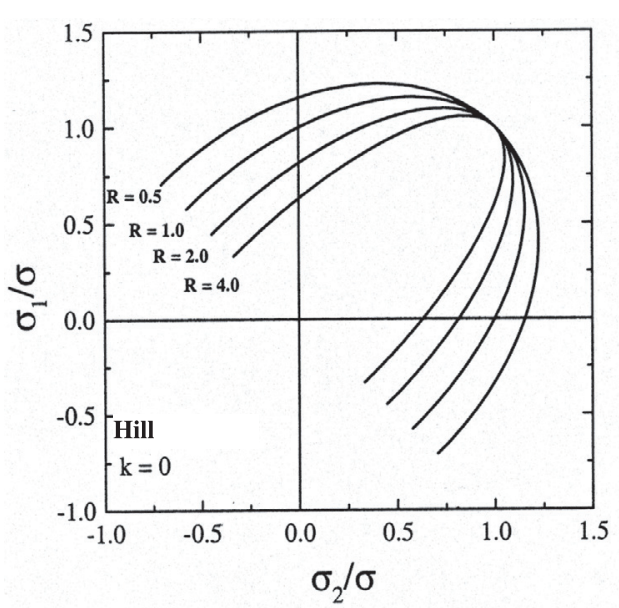

a)

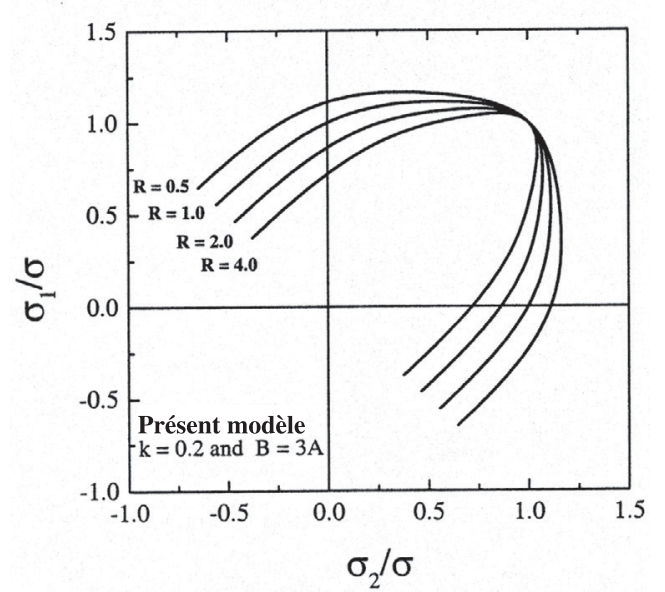

b)

Fig. 3. Surfaces de charge correspondant au critère quadratique de Hill pour différentes valeurs du coefficient de Lankford $R$.

un effet stabilisant est obtenu pour toute autre trajectoire, en raison de la déviation du chemin suivi dans la zone de sous-épaisseur vers la traction plane.

Nous comparons dans les simulations présentées ciaprès les résultats obtenus pour des matériaux pour lesquels les paramètres de la loi d'écrouissage sont définis en traction plane. Ainsi, la déformation équivalente limite $\tilde{\varepsilon}_{\mathrm{tp}}^{*}$ (identifiée en traction plane) ne dépend pas de la surface de charge considérée. Les résultats sont présentés dans le diagramme des déformations principales $\left(\varepsilon_{1}, \varepsilon_{2}\right)$ et ce pour des chemins de déformation linéaires.

\subsection{Influence de la forme de la surface de plasticité}

Différents matériaux modèles sont envisagés pour analyser l'influence de la surface de charge. Les surfaces de charge considérées correspondent au critère quadratique de Hill ( $k=0$ dans l'Éq. (16)) et au présent modèle (avec $k=0,2$ et $B=3 A$ dans (16)), et sont représentées respectivement sur les figures $3 a$ et $b$. 


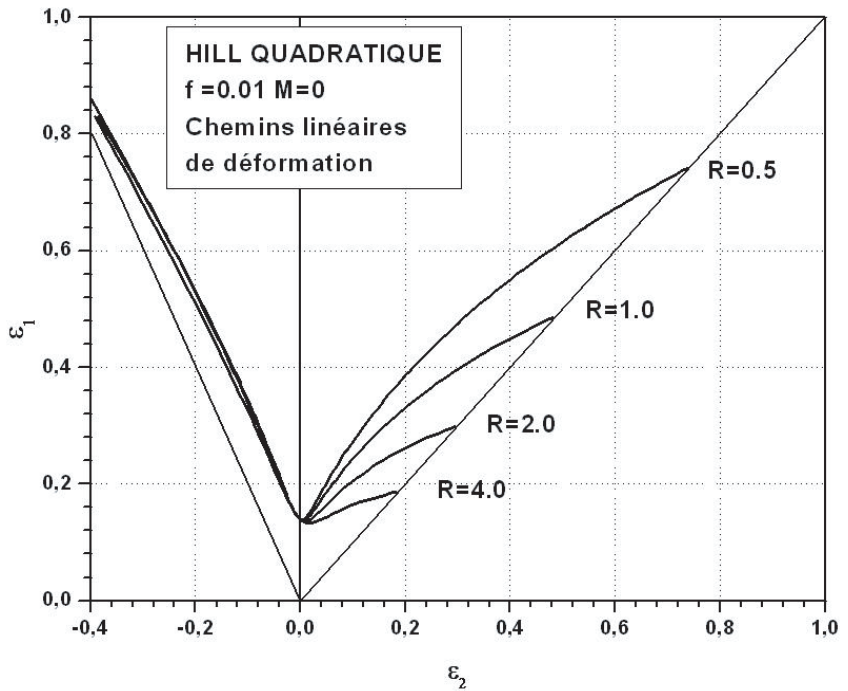

a)

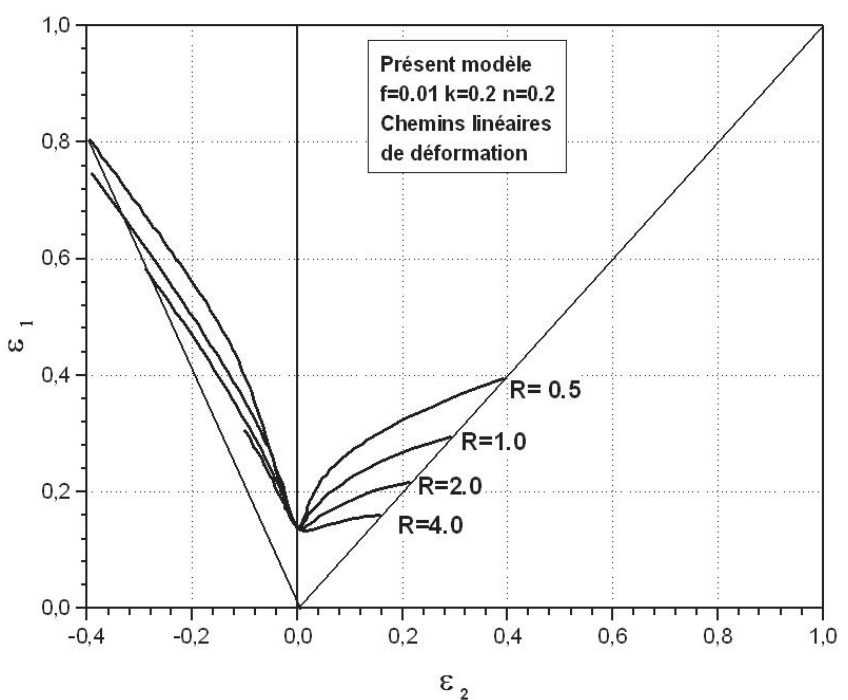

b)

Fig. 4. Courbes limites de formage pour des trajectoires linéaires et pour différentes surfaces de charge de la figure 3a correspondant au critère de Hill.

Les paramètres de la loi d'écrouissage sont obtenus par des essais de traction simple et des essais de traction plane.

Les limites correspondantes obtenues avec $f=0,01$, $N=0,2, \varepsilon_{0}=0,002$ et $M=0$, sont reportées sur les figures $4 \mathrm{a}$ et $\mathrm{b}$. Avec le critère quadratique de Hill (Fig. 3a), la courbure de la surface de charge dans le domaine de l'expansion croît (ou le rapport $P=\sigma_{\mathrm{tp}} / \sigma_{\mathrm{b}}$ entre la contrainte en traction plane et la contrainte en traction équibiaxiale décroît) lorsque le coefficient de Lankford $R$ croît, ce qui explique la diminution des déformations limites obtenues dans cette région lorsque $R$ croît (Fig. 4a). D'autre part le rapport $Q=\sigma_{\mathrm{tp}} / \sigma_{\mathrm{n}}$ entre la contrainte en traction plane et la contrainte en traction uniaxiale croît lorsque $R$ croît, ce qui explique

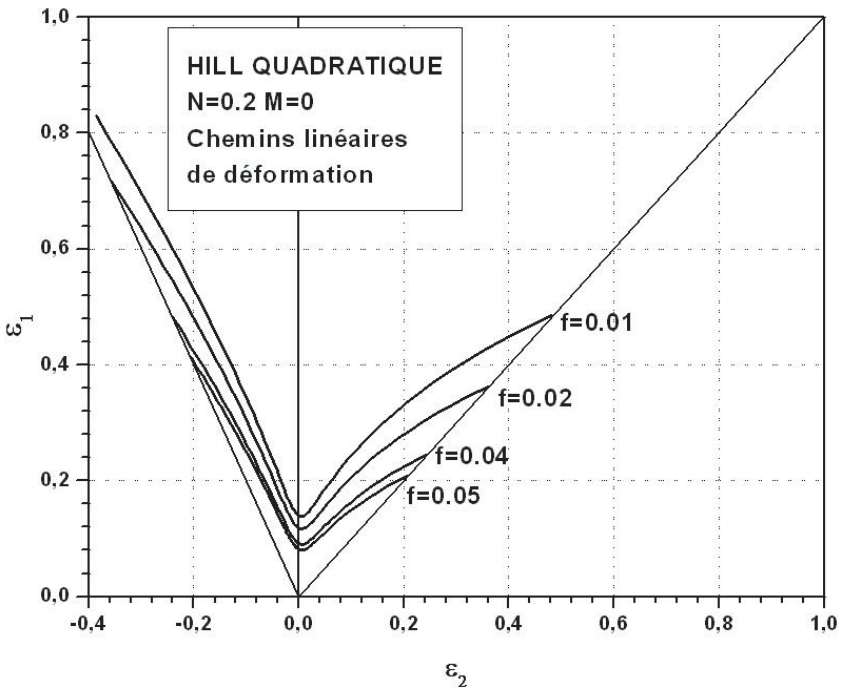

a)

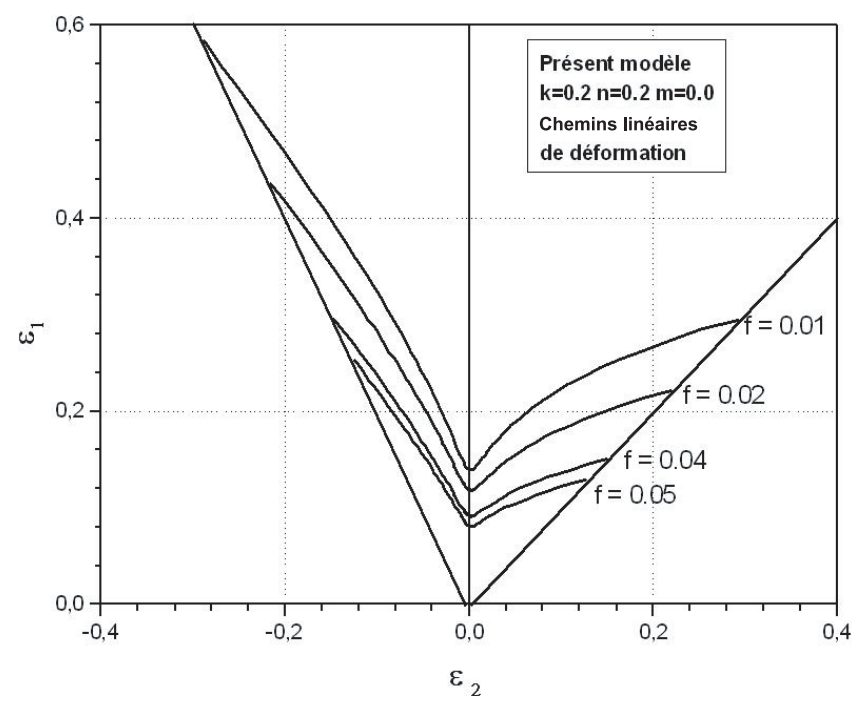

b)

Fig. 5. Influence de la taille du défaut sur les CLF selon le critère quadratique de Hill.

l'augmentation des déformations limites obtenues en rétreint lorsque $R$ croît, pour un rapport de contrainte donné. Ces tendances sont retrouvés avec le présent modèle, mais avec des valeurs de déformations limites moins élevées et une sensibilité moins marquée vis-à-vis de $R$ dans le domaine de l'expansion (Fig. 4b), en raison d'une plus faible valeur de $P$ pour un $R$ donné (Fig. $3 \mathrm{~b}$ )

\subsection{Influence de la taille du défaut, de l'exposant d'écrouissage et de la sensibilité à la vitesse de déformation}

Les influences de la taille du défaut $f$, de l'exposant d'écrouissage $N$ et du coefficient de sensibilité à la vitesse de déformation $M$ sont reportées sur les figures 5-7 pour un matériau de Von Mises insensible à la vitesse 


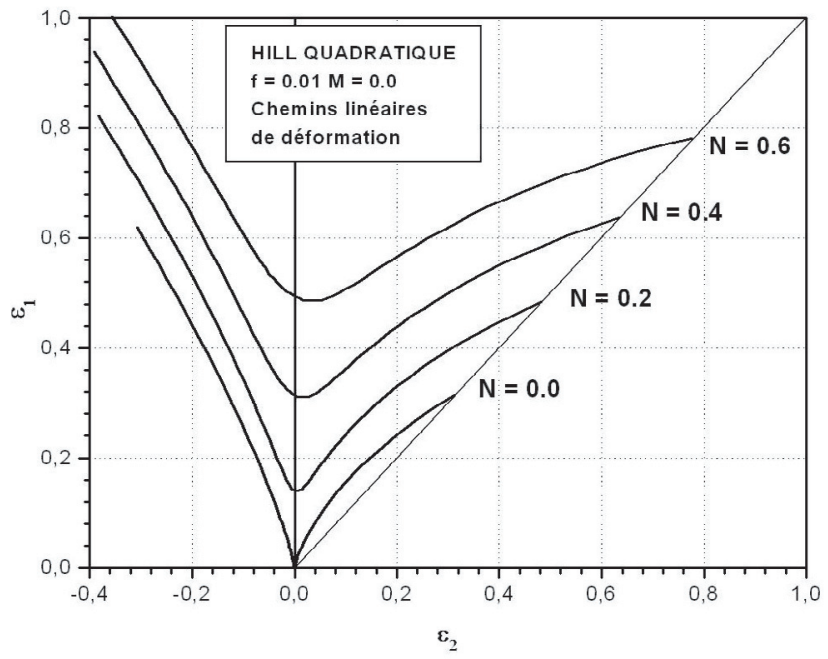

a)

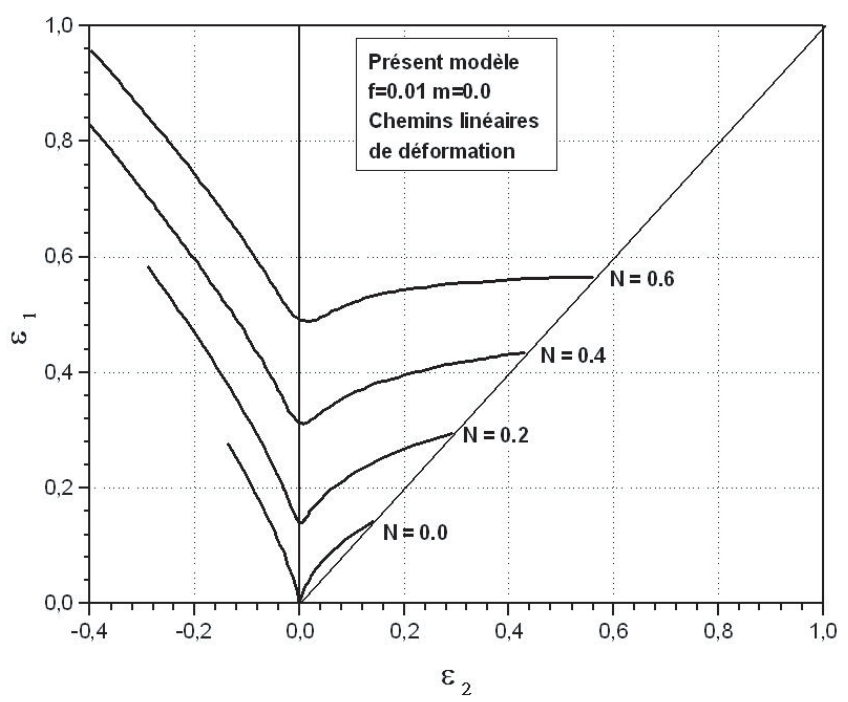

b)

Fig. 6. Influence de l'exposant d'écrouissage sur les CLF avec le critère quadratique de Hill a), avec le présent modèle b).

de déformation. Ces résultats montrent en particulier la très forte influence de la taille du défaut dans le domaine de l'expansion $(\rho>0)$, alors que les influences de $N$ et $M$ sont qualitativement comparables, et se traduisent par une élévation globale des limites de formage dans tout le domaine (de la traction simple à la traction équibiaxiale) lorsque $N$ et $M$ augmentent. On observe également dans le domaine du rétreint $(\rho\langle 0)$ que les déformations limites correspondent avec une bonne précision à une déformation en épaisseur critique indépendante du chemin de déformation $\left(\varepsilon_{1}+\varepsilon_{2}=\right.$ constante).

\subsection{Comparaison du modèle avec les résultats expérimentaux de la littérature}

Sur la figure 8, sont représentés les résultats obtenus par le modèle proposé, ceux obtenus avec le critère

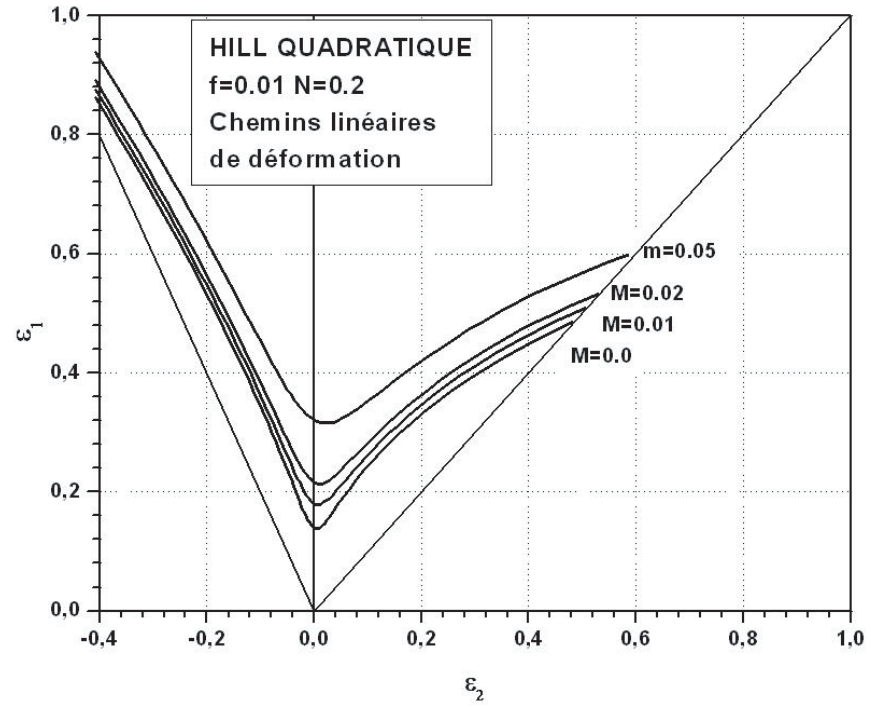

a)

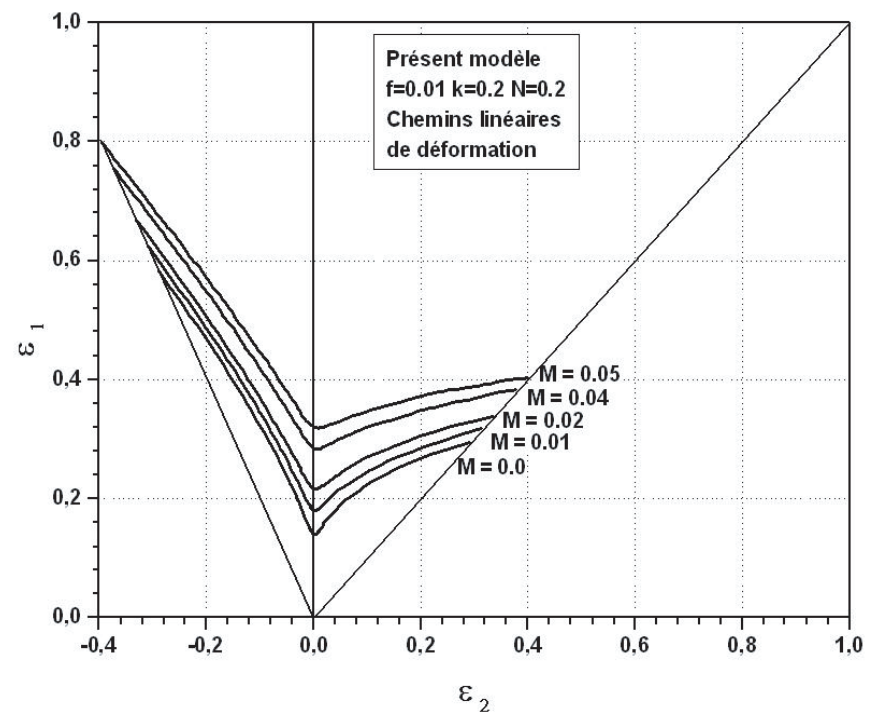

b)

Fig. 7. Influence du coefficient de sensibilité à la vitesse de déformation sur les CLF avec le critère quadratique de Hill a), avec le présent modèle b).

quadratique de Hill et les résultats expérimentaux réalisés par Graf et Hosford en 1994 [16]. Les essais sont relatifs à l'aluminium $\mathrm{Al}$ 6111-T4 dont les propriétés mécaniques et la loi de comportement sont représentés dans le tableau 2.

On constate que le modèle donne des résultats plus proches des résultats expérimentaux que ceux prévus par le critère de Hill, notamment dans le domaine $-0,1 \leq$ $\varepsilon_{2} \leq 0,1$. Par ailleurs pour $\varepsilon_{2}<-0,1$ et $\varepsilon_{2}>0,1$, on constate que le modèle donne des résultats nettement plus proches de l'expérience que ceux de Hill. En effet les résultats prévus par Hill s'éloignent nettement de ceux obtenus expérimentalement en particulier tout près de l'état de traction équibiaxiale. 


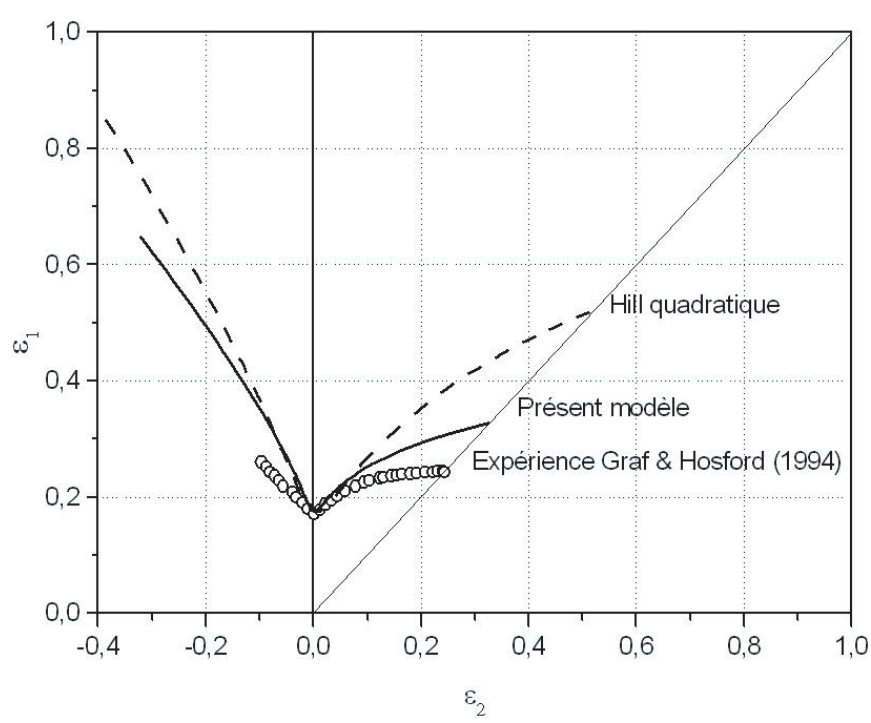

Fig. 8. CLF comparatives du modèle, de Hill et de l'expérience de Graf et Hosford [16].

Tableau 2. Caractéristiques mécaniques de l' Al 6111-T4

\begin{tabular}{ccc}
\hline $\begin{array}{c}\text { Angle par rapport à la } \\
\text { direction de laminage }\end{array}$ & $\begin{array}{c}\text { Limite élastique } \\
(\mathrm{MPa})\end{array}$ & $\begin{array}{c}\text { Valeurs du coefficient } \\
\text { de Lankford }\end{array}$ \\
\hline $0^{\circ}$ & 185 & 0,665 \\
$45^{\circ}$ & 169 & 0,63 \\
$90^{\circ}$ & 165 & 0,785 \\
Module de Young & \multicolumn{2}{c}{$70(\mathrm{GPa})$} \\
Coefficient de Poisson & 0,33 \\
Loi d'écrouissage & $\sigma=566,66(0,012+\varepsilon)^{0,2588}(\mathrm{MPa})$ \\
\hline
\end{tabular}

\section{Conclusion}

Dans ce travail l'utilisation d'un nouveau modèle de plasticité orthotrope en contraintes planes a conduit à la mise au point d'une analyse de localisation de la déformation visant à prédire l'apparition de la striction dans les tôles minces. Les simulations numériques effectuées pour des chemins de déformation linéaires ont permis d'analyser l'influence des paramètres du matériau sur les limites de formage des tôles. La confrontation entre les prévisions du programme et les résultats expérimentaux de la littérature a montré qu'un bon accord calcul/expérience pourrait être obtenu grâce à une prise en compte plus réaliste des caractéristiques de la surface d'écoulement.

\section{Références}

[1] Z. Marciniak, K. Kuczynski, Limit strains in the process of stretch-forming sheet metal, Int. J. Mech. Sci. 6 (1967) 609

[2] G. Ferron, R. Mahkouk, J. Morréale, A parametric description of orthotropic plasticity in metal sheet, Int. J. Plasticity 10 (1994) 431-449

[3] G. Ferron, A. Molinari, Mechanical and physical aspects of sheet metal ductility, FLD : Concepts, Methods and applications (1989) 111

[4] D.C. Drucker, Relation of experiments to mathematical theories of plasticity, J. Appl. Mech. 16 (1949) 349-360

[5] R. Hill, A theory of the yielding and plastic flow of anisotropic metals, Proc. Roy. Soc. London A 193 (1948) 281-297

[6] D. Banabic, E.Dannenmann, Prediction of the influence of yield locus on the limit strains in the sheet metals, J. Materials Processing Technology 109 (2001) 9-12

[7] M. Kuroda, V. Tvergaard, Forming limit diagrams for anisotropic metal sheets with different yield criteria, Int. J. Solids and Structures 37 (2000) 5037-5059

[8] N. Boudeau, J.C. Gelin, S. Salhi, Theoretical and numerical modelling of isotropic and anisotropic ductile damage in metal forming process in : SK Ghost, M Predeleanu (ed). Material processing defects, 1995, pp. 123-140

[9] N. Boudeau, J.C. Gelin, Necking in sheet metal forming. Influence of macroscopic properties of materials, Int. J. Mech. Sci. 42 (2000) 2209-2232

[10] F. Rondé Oustau, B. Baudelet, Microstructure and strain path in deep-drawing, Acta Metallurgica 25 (1997) $1523-1529$

[11] R. Mesrar, G. Ferron, Limits to ductility of metal sheets subjected to complex strain-paths, Int. J. Plasticity 14 (1998) 391-411

[12] R. Mesrar, Influence des paramètres rhéologiques du matériau sur l'emboutissage et étude des CLF, Thèse de doctorat d'état, Université Ibn Zohr, Agadir, Maroc, 1998

[13] T. Kawaba, S. Ikeda, K. Kuroda, Measurement and analysis of different work hardening in cold-rolled sheet under biaxial tension, J. Mater. Proc. Tech. 80/81 (1998) $517-523$

[14] T. Kawaba, I. Susuki, S. Ikeda, Identification of a yield locus of aluminium alloy sheet A5182-0 by biaxial tensile tests using cruciform specimens, JSTP 89 (1998) 56-61

[15] A. Zeghloul, R. Mesrar, G. Ferron, Analytical expression of the forming limits in biaxially stretched sheets, Int. J. Mech. Sci. 32 (1990) 981-990

[16] A. Graf, W.F. Hosford, The influence of strain path changes on forming limit diagrams of Al 6111-T4, Int. J. Mech. Sci. 36 (1994) 897 